\title{
National and provincial impact and cost- effectiveness of Haemophilus influenzae type $b$ conjugate vaccine in China: a modeling analysis
}

Haijun Zhang ${ }^{1,2+}$, Cristina Garcia ${ }^{3,4+}$, Wenzhou Yu ${ }^{5+}$, Maria Deloria Knoll ${ }^{3,4}$, Xiaozhen Lai ${ }^{1,2}$, Tingting Xu' ${ }^{6}$, Rize Jing ${ }^{1,2}$, Ying Qin ${ }^{7}$ Z Zundong Yin ${ }^{5^{*+}}$, Brian Wahl ${ }^{3,4^{*+}}$ and Hai Fang ${ }^{1,8,7^{*+}}$

\begin{abstract}
Background: Globally, Haemophilus influenzae type b (Hib) vaccine has substantially reduced the burden of Hib invasive disease. However, China remains the only country not to include Hib vaccine into its national immunization program (NIP), although it accounts for $11 \%$ of global Hib deaths. We aimed to assess the costeffectiveness of including Hib vaccine in China's NIP at the national and provincial levels.

Methods: Using a decision-tree Markov state transition model, we estimated the cost-effectiveness of Hib vaccine in the NIP compared to the status quo of Hib vaccine in the private market for the 2017 birth cohort. Treatment costs and vaccine program costs were calculated from Chinese Center for Disease Control and Prevention (CDC) and national insurance databases. Epidemiological data and other model parameters were obtained from published literature. Cases and deaths averted, quality-adjusted life years (QALYs) gained, and incremental cost-effectiveness ratios (ICER) were predicted by province. Deterministic and probabilistic sensitivity analyses were performed to explore model uncertainty.

Results: Including Hib vaccine in the NIP was projected to prevent approximately 2700 deaths (93\% reduction) and 235,700 cases of Hib disease (92\% reduction) for the 2017 birth cohort at the national level. Hib vaccine was costeffective nationally (US\$ 8001 per QALY gained) compared to the GDP per capita and cost-effective in 15 of 31 provinces. One-way and scenario sensitivity analyses indicated results were robust when varying model parameters, and in probabilistic sensitivity analysis, Hib vaccine had a 64\% probability of being cost-effective nationally.
\end{abstract}

\footnotetext{
*Correspondence: yinzd@chinacdc.cn; bwahl@jhu.edu; hfang@hsc.pku.edu.cn

+Haijun Zhang, Cristina Garcia, and Wenzhou Yu contributed equally as the first authors.

${ }^{5}$ National Immunization Program, Chinese Center for Disease Control and Prevention, Beijing, China

${ }^{3}$ Department of International Health, Johns Hopkins Bloomberg School of Public Health, Baltimore, USA

${ }^{1}$ China Center for Health Development Studies, Peking University, Beijing,

China

Full list of author information is available at the end of the article
}

(c) The Author(s). 2021 Open Access This article is licensed under a Creative Commons Attribution 4.0 International License, which permits use, sharing, adaptation, distribution and reproduction in any medium or format, as long as you give appropriate credit to the original author(s) and the source, provide a link to the Creative Commons licence, and indicate if changes were made. The images or other third party material in this article are included in the article's Creative Commons licence, unless indicated otherwise in a credit line to the material. If material is not included in the article's Creative Commons licence and your intended use is not permitted by statutory regulation or exceeds the permitted use, you will need to obtain permission directly from the copyright holder. To view a copy of this licence, visit http://creativecommons.org/licenses/by/4.0/ The Creative Commons Public Domain Dedication waiver (http://creativecommons.org/publicdomain/zero/1.0/) applies to the data made available in this article, unless otherwise stated in a credit line to the data. 
Conclusion: Introducing Hib vaccine in China's NIP is cost-effective nationally and in many provinces. Less socioeconomically developed provinces with high Hib disease burden and low access to Hib vaccine in the current private market, such as those in the west region, would benefit the most from adding Hib vaccine to the NIP. In the absence of a national policy decision on Hib vaccine, this analysis provides evidence for provincial governments to include Hib vaccine into local immunization programs to substantially reduce disease burden and treatment costs.

Keywords: Immunization, Haemophilus influenzae type b, China, Child health, Health economics

\section{Background}

Haemophilus influenzae type b (Hib) is a common cause of pneumonia, meningitis, and other serious infections in children $[1,2]$. China was estimated to be among the ten countries with the greatest number of Hib cases and deaths in children aged 1-59 months in 2000 [3]. Wahl et al. estimated that China still had approximately 3400 total Hib deaths in 2015 [4].

Vaccination is one of the most effective means of preventing Hib disease in a variety of settings around the world [5-7], and it remains an effective tool to reduce antibiotic resistance among some bacterial pathogens including Hib [8]. In September 2013, the World Health Organization (WHO) universally recommended the inclusion of Hib vaccines in all infant immunization programs worldwide, regardless of the availability of local or national surveillance data [2]. Hib vaccines have been introduced in 193 of 194 WHO member countries and regions [9]. China is the only WHO member that has not included Hib vaccine in its NIP. Rather, Hib vaccine is only available to children through the private market in China, where it first became available in 1996 [10]. While the global burden of Hib disease has decreased significantly in recent years with expanded access to Hib vaccine [11], China has a relatively large remaining burden of Hib disease [3, 4].

Policy decisions on introducing Hib vaccine into China's NIP have been driven by uncertainties around the burden of Hib disease without national surveillance and the high price of Hib vaccines [10]. A new law enacted in 2019 empowering provincial public health officers to make their own policies regarding new vaccine introductions presents the opportunity for provinces to introduce Hib vaccine before it is nationally introduced into the NIP [12]. High-quality studies on the economic impact of Hib vaccination in China are limited, and subnational analyses are not available. National and provincial data on the economic impact of Hib vaccination in China are needed to inform policy decisions about expanding the use of Hib vaccines. To address this evidence gap, we evaluated the national and provincial cost-effectiveness of introducing $\mathrm{Hib}$ vaccine into China's NIP compared to the status quo in the private market.

\section{Methods \\ Model overview}

A decision-tree Markov state transition model was developed to estimate the impact of Hib vaccine on disease burden, quality-adjusted life years (QALYs), and costs for the 2017 birth cohort in each province in China (Fig. 1). The model compared Hib vaccine introduction into the NIP and the status quo where Hib vaccine is only available in the private market. The model tracked Hib pneumonia, meningitis, and non-pneumonia nonmeningitis (NPNM) disease events over the cohort's first five years of life and estimated the QALYs gained over the life of the cohort. Children surviving past the neonatal period entered the model under both comparators (i.e., Hib vaccine in the NIP or status quo), and were assumed to be healthy but at risk of Hib infection depending on vaccination status (Fig. 1 and Additional file 1: Table S1).

The analysis was conducted from the societal perspective using a lifetime time horizon for the cohort. All costs and effects were discounted at $3 \%$ as recommended by the WHO [13], and all costs were converted to 2017 US dollars (1 US\$ $=6.8 \mathrm{RMB}$ ), adjusting for inflation when necessary [14]. The model was developed using TreeAge Pro 2020 (TreeAge Software, Inc., Williamstown, MA). Results were estimated nationally and for the 31 provinces in mainland China and three geographically contiguous and socioeconomically distinct regions: east, central, and west according to National Bureau of Statistics of China. Parameter point estimates, plausibility ranges, and distributions are presented in Table 1.

\section{Epidemiological data}

The province-specific probabilities of Hib severe and non-severe pneumonia, meningitis, and NPNM cases and deaths between ages 1 and 59 months were estimated from modeled provincial Hib incidence and mortality in 2017 [33] assuming no vaccination in the private market and following the Hib disease age distribution from published literature (Additional file 2: Table S2) [34]. We assumed all severe pneumonia, meningitis, and NPNM cases were hospitalized due to the severity of these syndromes, and all deaths occurred in hospitals. 

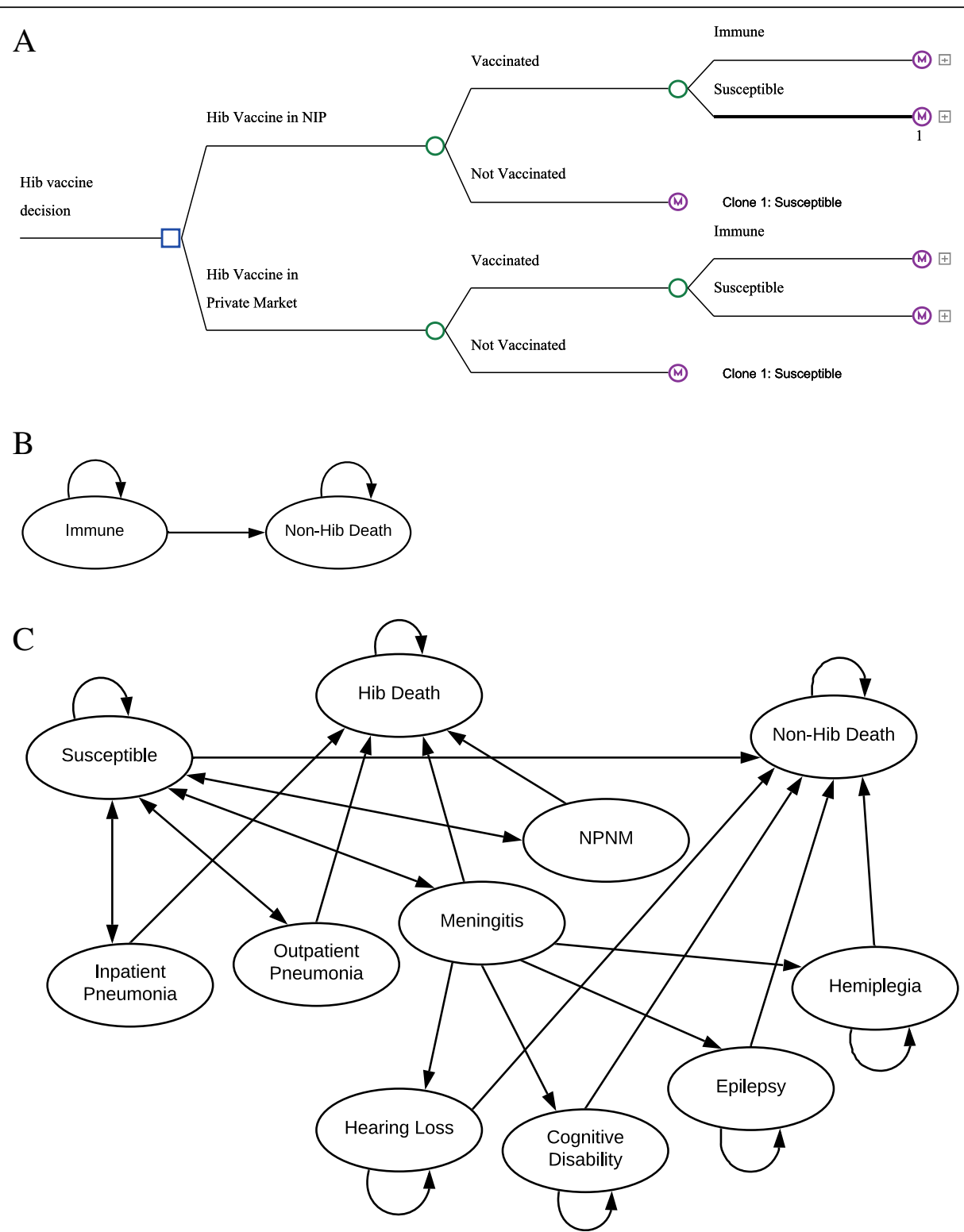

Fig. 1 Markov decision tree for a single birth cohort comparing Hib vaccine in the national immunization program (NIP) vs. Status quo (Hib vaccine in the private market). A Markov decision tree. B Hib Immune Markov diagram. C Hib susceptible Markov diagram

Access to care is high in most provinces, and most deaths of children under-five in China occur in healthcare facilities rather than in the community [1]. Children developing Hib meningitis in the model were at risk of long-term sequelae (i.e., cognitive disability, hearing loss, epilepsy, and hemiplegia) following probabilities from a global meta-analysis study [35].

\section{Disease burden costs}

Provincial and regional costs of Hib disease were estimated using syndrome-specific data from published literature and health insurance data from the China Healthcare Insurance Research Association (CHIRA) comprising data from hospitals in all 31 provinces in mainland China between 2013 and 2017 [18]. The cost per inpatient and outpatient case of pneumonia, meningitis, and NPNM included direct medical, direct nonmedical, and indirect costs. The cost of sequelae only included direct medical costs.

The average direct medical cost per case for each syndrome was estimated using the CHIRA individual-level medical cost data by ICD-10 code. Direct non-medical costs for inpatient pneumonia and meningitis, including the cost of transportation, accommodation, out-ofpocket medication, and other fees, were estimated from China CDC surveys conducted in 2015 in Gansu 


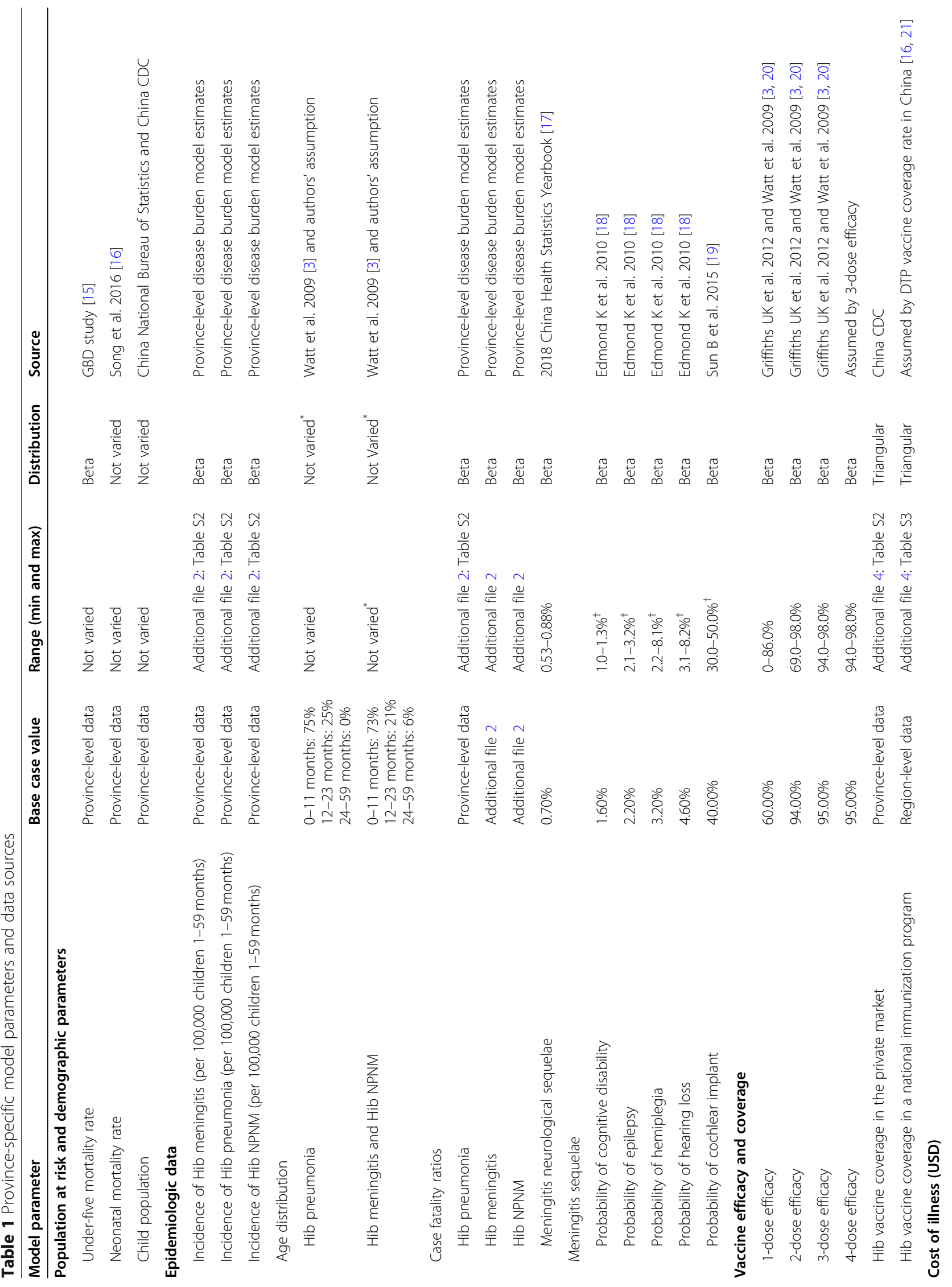




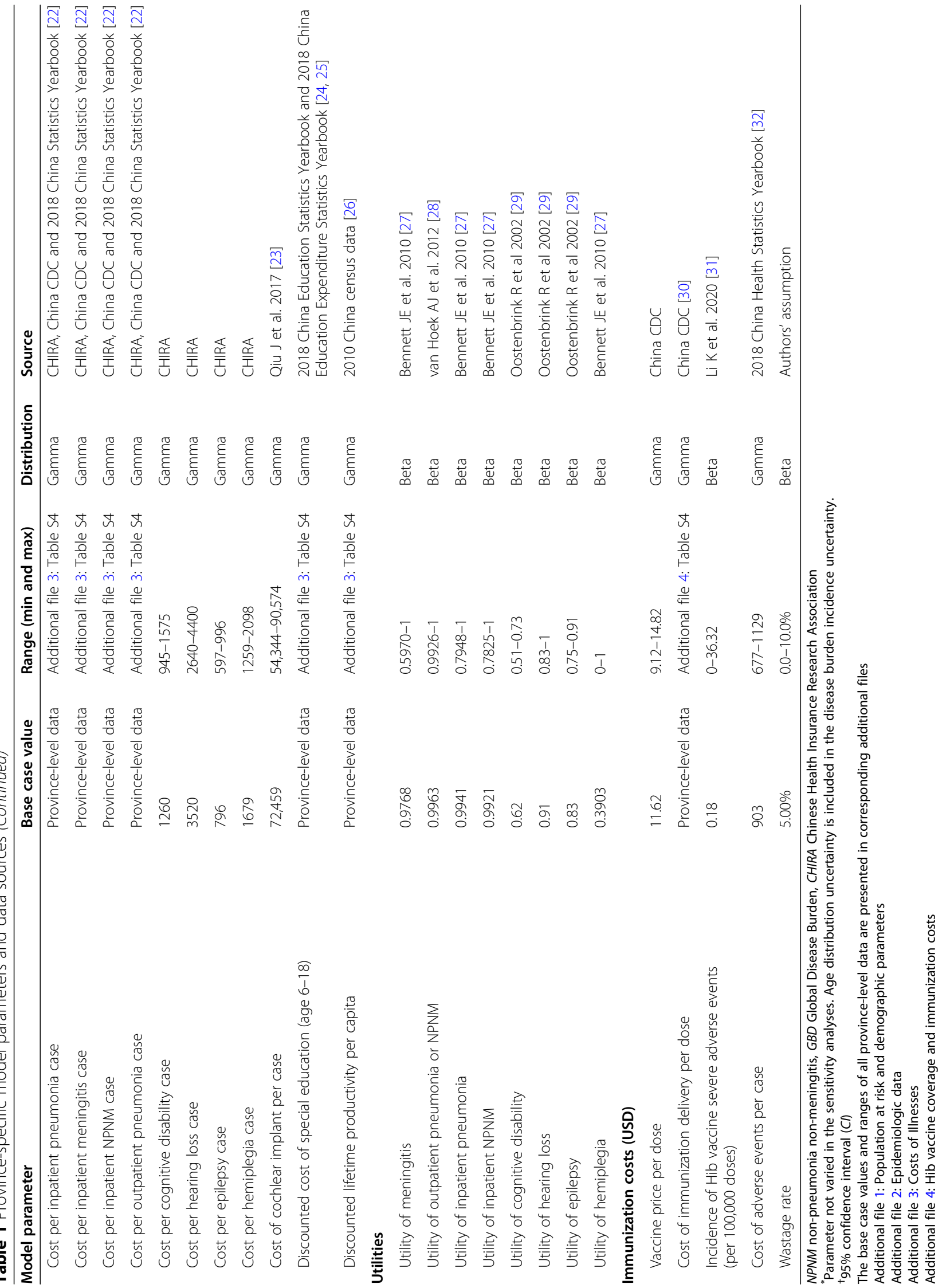


province [36] and 2014 in Shandong, Hebei, and Hubei provinces [37]. Estimates from these surveys were adjusted for each province using the ratio of the provincial total consumption expenditure obtained from the China Statistics Yearbook [22]. Because non-medical cost estimates for NPNM were not available, the cost was estimated using the average days hospitalized and the daily non-medical cost for inpatient pneumonia. Indirect costs associated with caregiver and visitor productivity loss and future lifetime productivity loss due to premature death and disability were estimated using the human capital approach. The methods used to estimate direct medical, non-medical costs, and indirect costs for each province are described in Additional file 3: Table S3 and S4.

\section{Vaccine efficacy and coverage rates}

Because a uniform Hib vaccine schedule does not exist in China, the base case modeled a $3+1$ dosing schedule assuming infants received primary doses by 6 months of age and a booster dose at the age of 18-24 months following the current schedule used in the private market and recommendations from the China CDC and vaccine manufacturers in China [38]. For the status quo scenario, provincial coverage rates for each dose of Hib vaccine in the private market were estimated by multiplying the total doses administered in each province from the China CDC by the distribution of children receiving 1,2 , 3 , and 4 doses obtained from a 2019 facility-based survey of more than 6000 children in 10 provinces in China (Additional file 4: Table S2) [3, 39]. For coverage in the NIP, regional four-dose diphtheria-tetanus-pertussis vaccine (DTP) coverage was used as a proxy for Hib vaccine coverage because of the similar schedule in China. Dosespecific vaccine efficacies were estimated from a metaanalysis study of controlled clinical trials globally [20]. Due to the relatively low coverage of Hib vaccine currently in China and the wide dispersion of immunized children, herd immunity was not included in the base case, but was included in the sensitivity analysis.

\section{Vaccination costs}

In China, several Hib vaccine products of varying prices are available in the private market. To estimate the cost of the vaccine in the private market, an average price per dose of US\$ 11.6 (range US\$ 9.1-14.9) was used based on centralized procurement data for seven Hib vaccine products in 2017 obtained from the China CDC [40]. For the base case analysis, the same vaccine price and schedule were used for both strategies to provide conservative results in the absence of guidance on vaccine introduction strategy from the Chinese government. The societal cost of the Hib vaccine program, including the governmental cost of routine immunization and the household cost of vaccine-seeking, was estimated using regional vaccine program data from a 2016 survey conducted by the China CDC in 15 provinces [30]. Governmental costs included the cost of vaccines, wastage, personnel, cold chain, surveillance, communication activities, training, and supervision at the national and provincial levels and the cost of serious adverse reaction. The vaccine-seeking costs included the cost of transportation and caregiver productivity loss. See Additional file 4 for the methods used to estimate the societal costs of the vaccine program for each strategy.

\section{Cost-effectiveness analysis}

Incremental cost-effectiveness ratios (ICERs), defined as the incremental costs (i.e., Hib vaccine costs and disease costs) per Hib case averted, death averted and QALY gained, were used to compare the status quo and NIP strategies. QALY utilities were derived from published literature [27-29] and ranged from 0 to 1 where 0 represented death and 1 represented perfect health (Table 1). The Chinese government has no policy for assessing cost-effectiveness thresholds of vaccines, so the costeffectiveness of Hib vaccine in the NIP was evaluated using two thresholds: (1) 2017 national and provincial GDP per capita thresholds as recommended by the Commission on Macroeconomics and Health [41]; and (2) thresholds estimated by Woods et al. for China (2017 US\$ 1130 and US\$ 4469), which account for the opportunity cost of the health expenditure and may be more appropriate to inform on resource allocation decisions [42]. The 2017 national GDP per capita was US\$ 8774, and provincial GDPs are described in Additional file 1: Table S1 [22].

\section{Sensitivity analysis}

Several sensitivity analyses were performed to test the robustness of model results and assess sources of model uncertainty. Deterministic sensitivity analyses (DSA) were conducted at the national level for all model parameters using the plausibility ranges specified in Table 1. For parameters with an unknown uncertainty range, the plausibility range was assumed to be $25 \%$ of the base value. Probabilistic sensitivity analysis (PSA) using Monte Carlo simulation ( $\mathrm{N}=1000$ iterations) was also done to assess the effects of changing multiple parameters simultaneously. Model uncertainty from the DSA and PSA were summarized using a tornado diagram and cost-effectiveness acceptability curves at the national level.

Scenario sensitivity analyses were performed to adjust the vaccine price per dose and vaccine schedules in the NIP and to include herd immunity. To estimate the influence of the NIP vaccine price on cost-effectiveness, we reduced the price of a Hib vaccine dose by $10-75 \%$ 
at national level. Although a $3+1$ schedule is currently used in the private market, no decision has been made on the schedule for the NIP. To ensure the Chinese government has sufficient data to evaluate $\mathrm{Hib}$ vaccine introduction, we estimated the impact of a 3-dose NIP schedule on the cost-effectiveness.

The combined direct and herd immunity effects at different vaccine coverage levels were estimated using regression estimates previously published by Wahl et al. [4]. When Hib vaccine coverage is $<10 \%$ or $\geq 98 \%$, the combined effect equals the direct effects. Because the assumed weighted vaccine coverage of all doses in the NIP strategy for all regions was more than $98 \%$, herd immunity would not likely have any effect on the NIP strategy. However, with variable weighted vaccine coverage in the private market, herd immunity could increase the effectiveness of the status quo strategy in some provinces. See Additional file 4 for the regression model used as well as provincial and regional weighted vaccine coverage estimates [4].

\section{Results}

\section{Impact of Hib vaccine}

Access and coverage of Hib vaccine in the private market varied substantially by the province in China. National coverage was only $33 \%$, ranging from over $50 \%$ in higher socioeconomically developed provinces like Shanghai and Tianjin to less than $5 \%$ in less socioeconomically developed provinces in the west region, like Tibet, Xinjiang, and Gansu.

The health effects and costs of introducing Hib vaccine into the NIP for the 2017 birth cohort are presented in Table 2. The model predicted that Hib vaccine in the NIP was projected to avert approximately $235,700 \mathrm{Hib}$ cases and 2700 Hib deaths, a 93\% reduction, over the first 5 years of life for the cohort. Most cases and deaths averted were pneumonia, with outpatient and inpatient pneumonia accounting for $80 \%$ and $17 \%$ of cases averted, respectively ( Additional file 5: Table S1). Guangdong and Hebei, the most populous provinces in China, had the greatest number of cases averted (i.e., > 10,000 cases). Most averted deaths were in Xinjiang and Yunnan provinces due to low Hib vaccine coverage and relatively high case fatality. The NIP strategy resulted in 85,388 QALYs gained over the cohort's lifetime with most QALYs gained in provinces in the west and poorer provinces like Xinjiang and Yunnan.

Introducing Hib vaccine into the NIP was estimated to cost US\$ 1.4 billion (US\$ 1.1 billion to the Chinese government) in vaccine procurement, programmatic costs, and indirect costs (Table 2 and Additional file 5: Table S2). However, investment in vaccination would be partially offset by savings of US\$ 377 million from averted treatment costs and increased lifetime productivity nationally.

\section{Cost-effectiveness analysis}

The national ICERs per case averted, per death averted, and per QALY gained were US\$2899, US \$252,686, and US\$ 8001, respectively. ICERs per QALY gained were less than the national GDP per capita (US\$ 8774) in 2017, indicating Hib vaccine in the NIP was highly costeffective.

At the provincial level, adding Hib vaccine to the NIP was cost-effective in 15 and 11 of the 31 provinces when compared to the provincial GDP per capita and Woods et al. threshold (US\$ 4,469), respectively (Fig. 2), and it was cost-saving in Qinghai, Tibet, and Xinjiang provinces-all in the west region. The provinces where Hib vaccination was not cost-effective had lower disease burden and higher vaccine coverage in the private market compared to the other provinces. Conversely, the three provinces where Hib vaccine was cost-saving had high Hib incidence, high CFRs, and lower vaccine coverage in the private market compared to other provinces (Additional file 6: Table S2).

\section{Sensitivity analysis}

In deterministic sensitivity analyses, Hib vaccination remained cost-effective when varying the model parameters (Fig. 3). The most important parameters were the price per dose in the NIP and disease burden parameters for Hib pneumonia and meningitis, including incidence and CFR. In the PSA, Hib vaccine in the NIP had a $64 \%$ probability of being cost-effective nationally compared to the national GDP per capita, and the probability increased to more than $80 \%$ when reducing the price of Hib vaccine by at least $10 \%$ (Fig. 4).

Including herd immunity in the model decreased the cost-effectiveness nationally and in most provinces. Herd immunity did not have any effect on the NIP strategy because of the high direct vaccine coverage in all provinces. However, accounting for herd immunity increased the vaccine effective coverage for the status quo strategy where the average provincial coverage in the private market was 33\%. More socioeconomically developed areas (e.g., Shanghai and Beijing) had higher vaccine coverage in the private market and greater herd immunity effects that decreased cost-effectiveness. Although the national ICER per QALY gained increased from US\$ 8001 to US\$14,903 when accounting for herd immunity, Hib vaccine in the NIP remained cost-effective in 9 provinces. It did not become cost-effective until the NIP vaccine price decreased by $50 \%$ or more. Nationally, adding Hib vaccine to the NIP became cost-saving when the price per dose was less than US\$ 2.02. A 3-dose schedule also increased cost-effectiveness overall (US\$ 


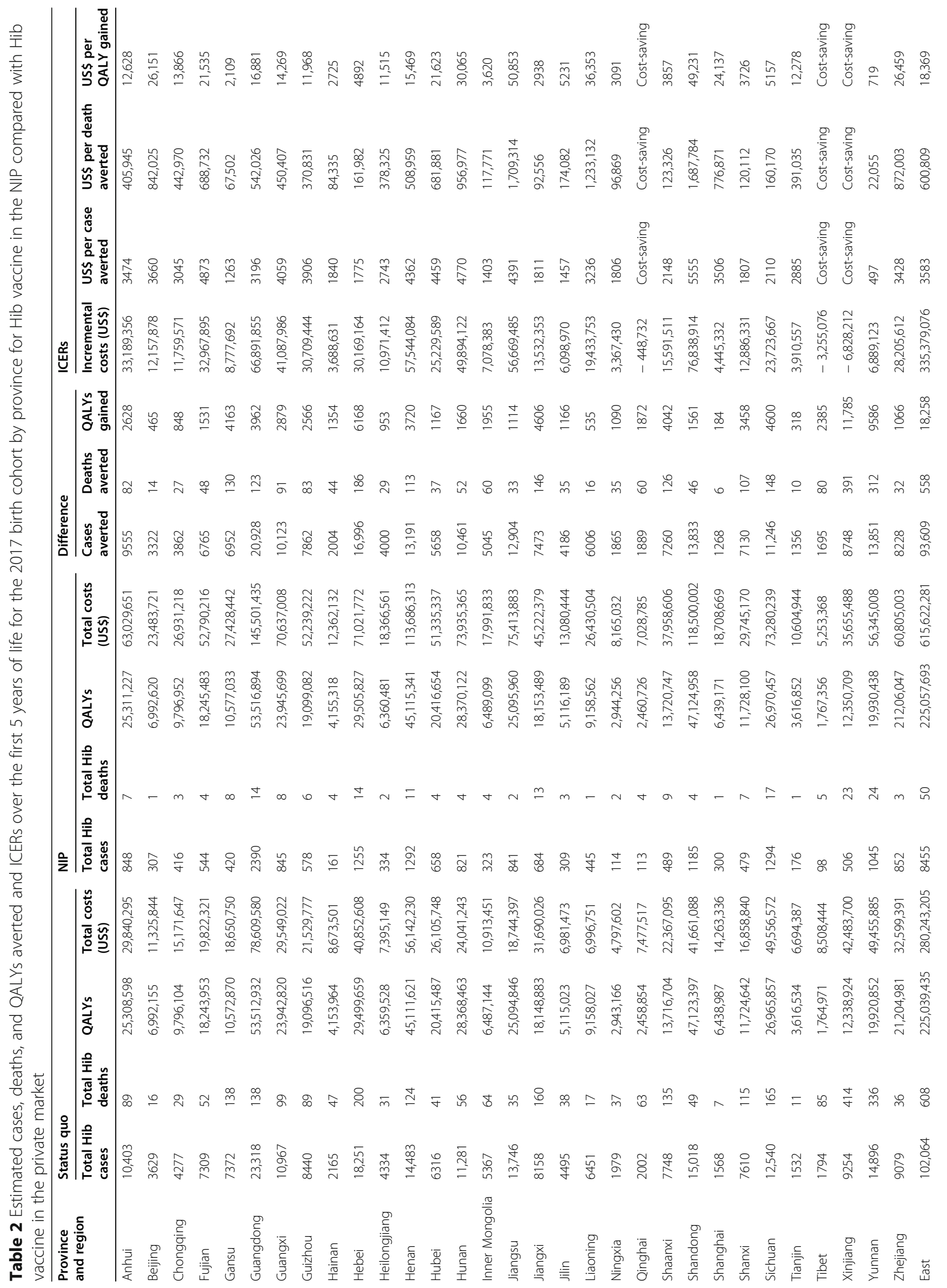




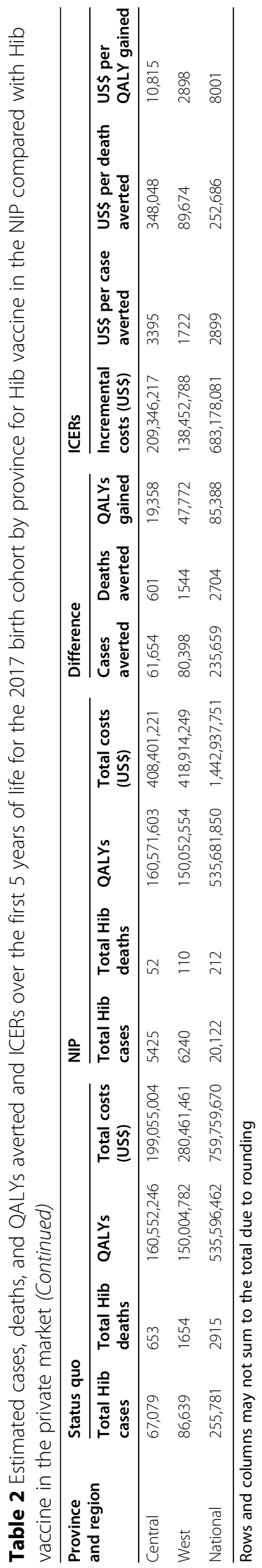




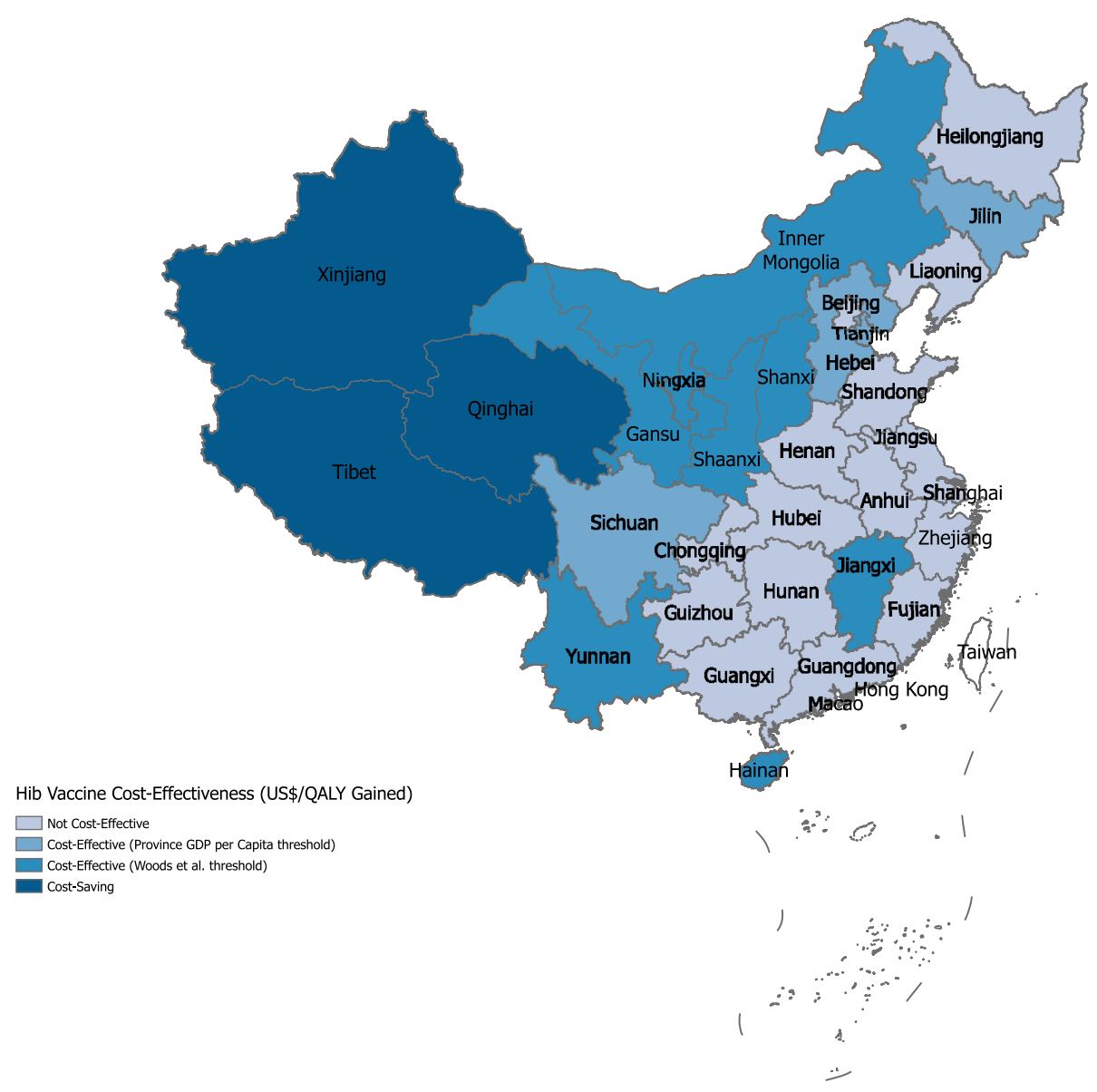

Fig. 2 Cost-effectiveness of Hib vaccine introduction in the national immunization program by province. The map indicates provinces where Hib vaccine in the national immunization program is cost-effective compared to the status quo when the ICER (US\$/QALY gained) is less than the provincial GDP per capita and the Woods et al. threshold for China (US\$ 4469). For Gansu province, the GDP per capita (US\$ 4313) is less than the Woods et al. threshold, and including Hib vaccine in the national immunization program is cost-effective at both thresholds

4071 per QALY gained nationally) with Hib vaccine in the NIP becoming cost-effective in 20 of 31 provinces (Additional file 7: Table S1).

\section{Discussion}

Currently, China is the only country not including Hib vaccine in its NIP, and continues to have the largest population of children without access to Hib vaccine despite availability in the private market. This is the first study at the provincial level to estimate the costeffectiveness of introducing Hib vaccine into the NIP compared to the status quo in the private market. For a single birth cohort in 2017, the introduction of Hib vaccine into the NIP could save approximately 2700 lives and avert over 235,600 cases of Hib invasive disease. Averting premature death and long-term disability from Hib sequelae resulted in savings of \$ 384 million in averted treatment costs and increased lifetime productivity from averted premature death. With an ICER per QALY gained of US\$ 8001, Hib vaccine in the NIP was cost-effective nationally compared to the 2017 GDP per capita. Adding Hib vaccine to China's NIP is not only cost-effective but would also expand access to the vaccine for children throughout China. Although this is the first study to compare national vaccination with the status quo in the private market, our findings at the national level are consistent with other studies comparing national vaccination to no vaccination $[43,44]$.

At the provincial level, Hib vaccine in the NIP was cost-effective in 15 of 31 provinces with provinces in the west region getting the largest benefit. Expanded Hib vaccination was most cost-effective in provinces with low coverage in the private market and/or higher Hib disease burden. While other programs and interventions aimed at improving maternal and child health had been implemented throughout China in the last decade [45, 46], none of these prevented Hib disease, and in less socioeconomically developed provinces, mostly in the west region, Hib disease burden remained high while access to vaccines in the private market was limited. In 


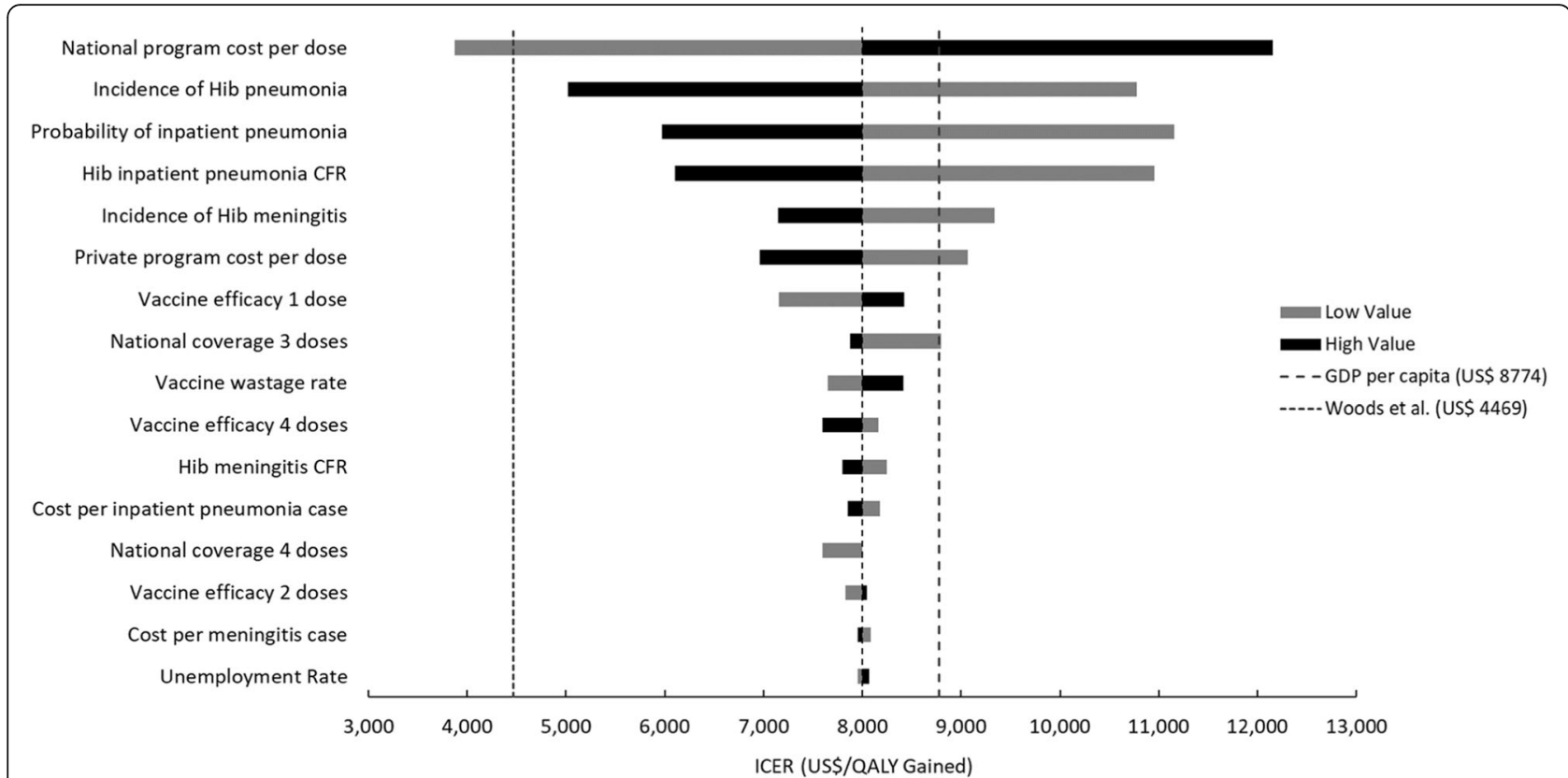

Fig. 3 Tornado diagram of one-way sensitivity analyses for the most influential model parameters on ICER (US\$/QALY gained)

Qinghai, Tibet, and Xinjiang, all provinces with low Hib vaccine coverage in the private market and high mortality, introducing Hib vaccine into these provinces was not only cost-effective but also cost-saving. Introducing Hib vaccine into China's NIP could not only effectively reduce the disease burden especially in the west region, but it would also promote health equity by improving vaccine access in less socioeconomically developed and higher disease burden provinces.

For the base case analysis, we used a conservative approach to estimate model parameters, but the sensitivity analyses demonstrated the robustness of the study results. Vaccine price, incidence, and CFR were among the main drivers of cost-effectiveness. In the absence of reliable vaccine price data in China, which was not Gavi eligible, we assumed the NIP vaccine price was the same as the private market. The Chinese government is likely to negotiate a reduced price for Hib vaccines when purchasing a very large volume per year if Hib vaccine is added to the NIP. Nationally, Hib vaccine, which was already cost-effective in the base case analysis, would become cost-saving if the vaccine price could drop from US\$ 11.62 to US\$ 2.02. Regionally, Hib vaccination became cost-effective in all three regions at US\$ 5.82.

This study had some limitations. First, reliable data on access to care for each province and deaths occurring outside health facilities were unavailable. Nationally, access to care was high in China, and we assumed all Hib cases sought care at a health facility and all deaths occurred in hospitals. Reductions in care-seeking would reduce cost savings and increase ICER estimates.
Conversely, deaths occurring outside health facilities could result in an underestimate of ICER values. Second, the model did not account for the dispersion of children vaccinated in the private market in the community. We assumed coverage was equally distributed throughout the province, but in reality, there are likely pockets of unvaccinated children and pockets with higher vaccine coverage where indirect effects could be present.

Third, the analysis relied on treatment cost data from CHIRA that has some limitations. While CHIRA cost data were nationally representative, it might overestimate the cost of Hib-related disease because data came from mostly urban areas and might not represent the rural populations. This urban bias might result in an underestimate of the ICERs in provinces with large rural populations and overestimate the cost-effectiveness. Despite this limitation, the sensitivity analysis showed that the vaccine price and disease burden parameters were the largest drivers of the ICER estimates, and varying in the cost of treatment was not likely to change the costeffectiveness in individual provinces. Similarly, the cases included in the CHIRA database included both laboratory-confirmed and suspected cases that could bias the treatment cost estimates. However, in China, high antibiotic use makes it difficult to laboratoryconfirm Hib cases, and most laboratories do not detect the majority of Hib disease. Due to the low confirmation rates in China, the treatment of confirmed and nonconfirmed cases of suspected bacterial origin was similar, and non-confirmed cost data were not likely to significantly affect the overall treatment cost estimates. 


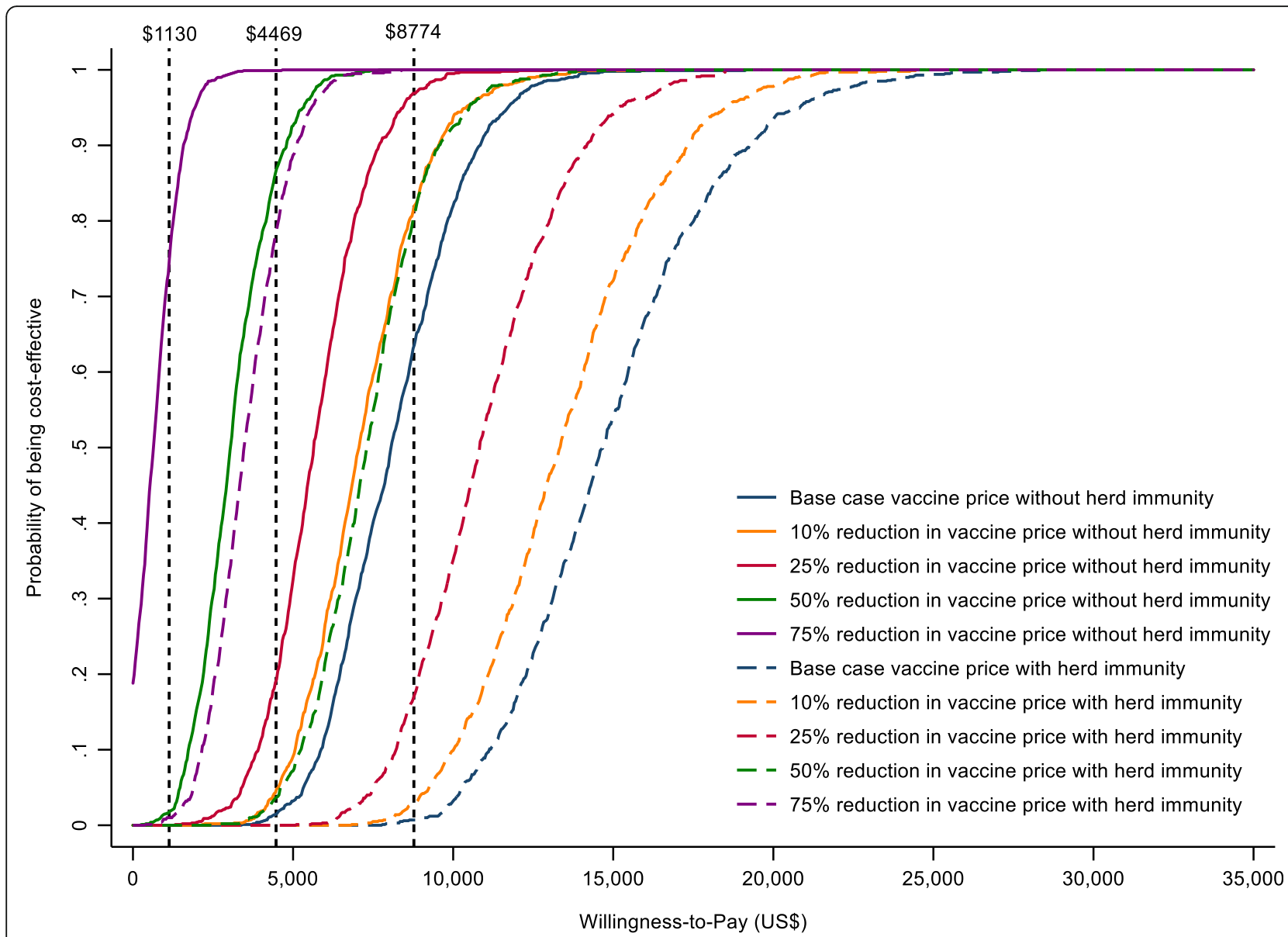

Fig. 4 Cost-effectiveness acceptability curves of the national Hib vaccine program for the base case and different vaccine price and herd immunity scenarios. Vertical lines represent the national GDP per capita (US\$ 8774), upper bound threshold (US\$ 4469) by Woods et al. (2016), and lower bound threshold (US\$ 1130) by Woods et al. (2016). The probability that adding Hib vaccine into the National Immunization Program is cost-effective for the base case and when reducing the price of Hib vaccine and accounting for the herd immunity from the probabilistic sensitivity analysis (PSA)

Fourth, incidence and treatment costs of Hib sequelae were not available by province, so national estimates obtained from published literature were used in the model. The deterministic sensitivity analysis showed that the impact of Hib sequelae parameters was minimal compared to other parameters, but this could be an underestimate because the treatment cost estimates used did not account for lifelong costs to treat the sequelae.

\section{Conclusions}

Despite these limitations, Hib vaccine was costeffective at the national level, in several provinces in China, and even cost-saving in three west provinces. This study provides evidence to support the introduction of Hib vaccine into China's NIP. Although Hib vaccine was available in the private market, the majority of children, especially those in poorer and higher burden areas, still lacked access because of the high price of Hib vaccines. The provincial analyses supported subnational introduction of Hib vaccine if no decision was made nationally, and priority should be given in provinces of Tibet, Xinjiang, and Qinghai due to their severe disease burden and substantial benefits gained from including Hib vaccine in their local immunization programs. Introduction of $\mathrm{Hib}$ vaccine in the NIP or in high burden provinces should be a key strategy to meet the Sustainable Development Goal child survival targets by 2030 and accelerate the elimination of Hib diseases globally.

\section{Abbreviations}

Hib: Haemophilus influenzae type b; NIP: National immunization program; CDC: Center for disease control and prevention; WHO: World health organization; ICER: Incremental cost-effectiveness ratios; QALY: Qualityadjusted life year; NPNM: Non-pneumonia non-meningitis; CHIRA: China healthcare insurance research association; GDP: Gross domestic product; DSA: Deterministic sensitivity analyses; PSA: Probabilistic sensitivity analysis 


\section{Supplementary Information}

The online version contains supplementary material available at https://doi. org/10.1186/s12916-021-02049-7.

Additional file 1: Table S1- Model live birth cohort and mortality rate parameters by province

Additional file 2: Table S2- Province-specific disease burden model parameters and data sources; Table S3- Hib disease burden among children 1-59 months by province.

Additional file 3: Table S4- ICD-10 codes used for each syndrome; Table S5- Summary of individual-level cost data from 2013 to 2017 obtained from the China Healthcare Insurance Research Association (CHIRA); Table S6- Inputs for the calculations of indirect costs (US\$); Table S7Cost of illness and lifetime productivity estimates (US\$).

Additional file 4: Table S8- Summary of Hib vaccine products available in China; Table S9- Provincial vaccine coverage and weighted vaccine coverage in the private in 2017; Table S10- Regional dose-specific and weighted combined Hib vaccine coverage used for the NIP strategy; Table S11- Societal cost per dose of the National Immunization Program (NIP) in China in 2015 (2017 US\$).

Additional file 5: Table S12- Provincial disease burden and economic supplemental results; Table S13- Provincial discounted economic costs of Hib disease and vaccine program costs (2017 US\$) for each vaccination strategy from the societal perspective.

Additional file 6: Table S14- Incremental cost-effectiveness ratios of including Hib vaccine in the NIP in 2017 (RMB); Table S15- Incremental cost-effectiveness ratios of including Hib vaccine in the NIP in 2017 (US\$); Table S16- Hib cases and deaths averted by province and region when including herd immunity effects.

Additional file 7: Table S17- Incremental cost-effectiveness ratios (US\$) for the different Hib vaccine schedules (4 doses vs. 3 doses in the NIP); Table S18- National incremental cost-effectiveness ratios (US\$ per QALY gained) when varying the NIP Hib vaccine price; Table S19- Provincial incremental cost-effectiveness ratios (US\$ per QALY gained) when reducing the NIP Hib vaccine price.

\section{Acknowledgements}

Haijun Zhang, Cristina Garcia, and Wenzhou Yu contributed equally as the co-first authors. Zundong Yin, Brian Wahl, and Hai Fang contributed equally as the corresponding authors. We thank the Chinese Health Insurance Research Association (CHIRA) to provide us the national health insurance data.

\section{Authors' contributions}

HF proposed and initiated this study. $\mathrm{HZ}, \mathrm{CG}$, and WY did the analyses for vaccine cost-effectiveness and wrote the first draft of the manuscript. MDK, $B W, Z Y$, and HF designed the project and oversaw the analysis and manuscript writing. $H Z, X L, T X, Y Q, R J$, and WY prepared provincial vaccine coverage and population data in China. HF supervised the entire project and acquired funding. All co-authors provided feedback during the design and interpretation of the project. They also contributed to revisions of the manuscript. All authors read and approved the final manuscript.

\section{Funding}

This work was supported, in whole or in part, by the Bill \& Melinda Gates Foundation [Grant Number: OPP1216660]. Under the grant conditions of the Foundation, a Creative Commons Attribution 4.0 Generic License has already been assigned to the Author Accepted Manuscript version that might arise from this submission. The sponsors played no role in study design, data collection, data analysis, data interpretation, or writing of the report.

\section{Availability of data and materials}

The datasets generated and analyzed during the current study are available from the corresponding author (Hai Fang) on reasonable request.

\section{Declarations}

Ethics approval and consent to participate Not applicable.

\section{Consent for publication}

Not applicable.

\section{Competing interests}

Hai Fang reports grants from the Bill \& Melinda Gates Foundation. Brian Wahl reports grants from the Bill \& Melinda Gates Foundation; Gavi, the Vaccine Alliance. Maria Deloria Knoll reports grants from the Bill \& Melinda Gates Foundation and Merck, and personal fees from Merck, Novartis, and Pfizer, outside of the submitted work. Cristina Garcia reports grants from the Bill \& Melinda Gates Foundation, Gavi, the Vaccine Alliance, and personal fees from Merck outside of the submitted work. All other authors declare no competing interests.

\section{Author details}

${ }^{1}$ China Center for Health Development Studies, Peking University, Beijing, China. ${ }^{2}$ Department of Health Policy and Management, School of Public Health, Peking University, Beijing, China. ${ }^{3}$ Department of International Health, Johns Hopkins Bloomberg School of Public Health, Baltimore, USA. ${ }^{4}$ International Vaccine Access Center, Johns Hopkins Bloomberg School of Public Health, Baltimore, USA. ${ }^{5}$ National Immunization Program, Chinese Center for Disease Control and Prevention, Beijing, China. ${ }^{6}$ Department of Health Management and Policy, School of Public Health, Capital Medical University, Beijing, China. ${ }^{7}$ Division of Infectious Diseases, Chinese Center for Disease Control and Prevention, Beijing, China. ${ }^{8}$ Peking University Health Science Center, Chinese Center for Disease Control and Prevention Joint Research Center for Vaccine Economics, Beijing, China. ${ }^{9}$ Key Laboratory of Reproductive Health, National Health Commission of the People's Republic of China, Beijing, China.

Received: 13 March 2021 Accepted: 30 June 2021

Published online: 11 August 2021

\section{References}

1. He C, Liu L, Chu Y, Perin J, Dai L, Li X, et al. National and subnational allcause and cause-specific child mortality in China, 1996-2015: a systematic analysis with implications for the Sustainable Development Goals. Lancet Glob Health. 2017;5(2):e186-97

2. World Health Organiztion. Haemophilus influenzae type b (Hib) Vaccination WHO position paper: July 2013-Recommendations. Vaccine. 2013;31(52):6168-9.

3. Watt JP, Wolfson LJ, O'Brien KL, Henkle E, Deloria-Knoll M, McCall N, et al. Burden of disease caused by Haemophilus influenzae type $b$ in children younger than 5 years: global estimates. Lancet. 2009;374(9693):903-11.

4. Wahl B, O'Brien KL, Greenbaum A, Majumder A, Liu L, Chu Y, et al. Burden of Streptococcus pneumoniae and Haemophilus influenzae type $b$ disease in children in the era of conjugate vaccines: global, regional, and national estimates for 2000-15. Lancet Glob Health. 2018;6(7):e744-57.

5. Gupta M, Prinja S, Kumar R, Kaur M. Cost-effectiveness of Haemophilus influenzae type $b(\mathrm{Hib})$ vaccine introduction in the universal immunization schedule in Haryana State, India. Health Policy Plan. 2013;28(1):51-61.

6. Akumu AO, English M, Scott JA, Griffiths UK. Economic evaluation of delivering Haemophilus influenzae type b vaccine in routine immunization services in Kenya. Bull World Health Organ. 2007;85(7):511-8.

7. Cochi SL, Broome CV, Hightower AW. Immunization of US children with hemophilus influenzae type b polysaccharide vaccine: a cost-effectiveness model of strategy assessment. JAMA. 1985;253(4):521-9.

8. Klugman KP, Black S. Impact of existing vaccines in reducing antibiotic resistance: Primary and secondary effects. Proc Natl Acad Sci. 2018;115(51):12896-901.

9. International Vaccine Access Center, Johns Hopkins Bloomberg School of Public Health. VIEW-hub-Hib vaccine. Baltimore: IVAC; 2021. http://www. view-hub.org. Accessed 10 Feb 2021.

10. Yu H, Yang W, Varma JK. To save children's lives, China should adopt an initiative to speed introduction of pneumonia vaccines. Health Aff (Millwood)0.2012; 31(11):2545-2553.

11. Levine $O S$, Knoll MD, Jones A, et al. Global status of Haemophilus influenzae type $b$ and pneumococcal conjugate vaccines: evidence, policies, and introductions. Curr Opin Infect Dis. 2010;23(3):236-41.

12. The National People's Congress of the People's Republic of China. Vaccine Administration Law of the People's Republic of China. 2019. http://www. npc.gov.cn/npc/c30834/201907/11447c85e05840b9b12c62b5b645fe9d. shtml. Accessed 3 May 2021. 
13. World Health Organiztion. WHO guide for standardization of economic evaluations of immunization programmes. 2nd ed. Geneva, Switzerland: WHO; 2019. https://www.who.int/immunization/documents/who_ivb_19.10/en/

14. World Bank. Official exchange rate. Washington: World Bank; 2021. https://data.worldbank.org/indicator/PA.NUS.FCRF. Accessed 11 Jan 2021.

15. Zhou M, Wang H, Zeng X, Yin P, Zhu J, Chen W, et al. Mortality, morbidity, and risk factors in China and its provinces, 1990-2017: a systematic analysis for the Global Burden of Disease Study 2017. Lancet. 2019;394(10204):1145-58.

16. Song P, Theodoratou E, Li X, Liu L, Chu Y, Black RE, et al. Causes of death in children younger than five years in China in 2015: an updated analysis. J Glob Health. 2016:6(2):020802.

17. Wang Y, Li X, Zhou M, Luo S, Liang J, Liddell CA, et al. Under-5 mortality in 2851 Chinese counties, 1996-2012: a subnational assessment of achieving MDG 4 goals in China. Lancet. 2016;387(10015):273-83.

18. Chinese Health Insurance Research Association. Chinese Health Insurance Research Association website. Beijing: CHIRA; 2021. https://www.zgylbx. com/. Accessed 14 Jan 2021.

19. Sun B. Investigation on neonatal hearing screening status in Zhengzhou, 2009-2013. Matern Child Health Care China. 2015;30(4):571-3.

20. Griffiths UK, Clark A, Gessner B, Miners A, Sanderson C, Sedyaningsih ER, et al. Dose-specific efficacy of Haemophilus influenzae type b conjugate vaccines: a systematic review and meta-analysis of controlled clinical trials. Epidemiol Infect. 2012;140(8):1343-55.

21. Cao L, Wang HQ, Zheng JS, Yuan P, Cao LS, Zhang GM. National Immunization Coverage Survey in China after Integrated more Vaccines into EPI Since 2008. Chin J Vaccines And Immunization.2012; 18(05):419-424+478.

22. National Bureau of Statistics of People's Republic of China. China Statistics Yearbook 2018. Beijing: China Statistics Press; 2018.

23. Qiu J, Yu C, Ariyaratne TV, Foteff C, Ke Z, Sun Y, et al. Cost-Effectiveness of Pediatric Cochlear Implantation in Rural China. Otology \& Neurotology. 2017:38(6):75-84.

24. Ministry of Education of the People's Republic of China. China Education Statistics Yearbook 2018. Beijing: China Statistics Press; 2018. p. 176-182.

25. Ministry of Education of the People's Republic of China. China Education Expenditure Statistical Yearbook 2018. Beijing: China Statistics Press; 2018.

26. National Bureau of Statistics of People's Republic of China. Tabulation on the 2010 population census of the People's Republic of China. http://www. stats.gov.cn/tjsj/pcsj/rkpc/6rp/indexch.htm. Accessed 20 Jan 2021.

27. Bennett JE, Sumner W, Downs SM, Jaffe DM. Parents' utilities for outcomes of occult bacteremia. Arch Pediatr Adolesc Med. 2000;154(1):43-8.

28. Van Hoek AJ, Choi YH, Trotter C, Miller E, Jit M. The cost-effectiveness of a 13-valent pneumococcal conjugate vaccination for infants in England. Vaccine. 2012;30(50):7205-13.

29. Oostenbrink R, Moll HA, Essink-Bot M-L. The EQ-5D and the Health Utilities Index for permanent sequelae after meningitis: a head-to-head comparison. J Clin Epidemiol. 2002;55(8):791-9.

30. Yu W, Lu M, Wang H, Rodewald L, Ji S, Ma C, et al. Routine immunization services costs and financing in China, 2015. Vaccine. 2018;36(21):3041-7.

31. Li K, Zhang L, Ye J, Ji S, Yu W, Cao L. Surveillance of adverse events following immunization in China, 2017. Chin J Vaccines Immunization. 2020:26(1):9-18.

32. National Health and Family Planning Commission of China. China Health and Family Planning Statistical Yearbook 2018. Beijing: Chinese Academy of Medical Sciences \& Peking Union Medical College Press; 2018.

33. Lai XZ, Wahl B, Yu WZ, Xu T, Zhang HJ, Garcia C, Guo Y, Yin ZD, Knoll MD, Fang $\mathrm{H}$. National, regional, and provincial disease burden associated with Streptococcus pneumoniae and Haemophilus influenzae type b in children in China: modelled estimates for 2010-17. 2021.(Under review)

34. Gupta M, Kumar R, Deb AK, Bhattacharya SK, Bose A, John J, et al. Multi-center surveillance for pneumonia \& meningitis among children $(<2$ yr) for Hib vaccine probe trial preparation in India. Indian J Med Res. 2010;131:649-58.

35. Edmond K, Clark A, Korczak VS, Sanderson C, Griffiths UK, Rudan I. Global and regional risk of disabling sequelae from bacterial meningitis: a systematic review and meta-analysis. Lancet Infect Dis. 2010;10(5):317-28.

36. Ning GJ, Wang XX, Liu SW, Zhu YY, Zhang BL, Zhang XS. Retrospective investigation on diseases burden of children with community acquired pneumonia under 5 years in Baiyin City of Gansu Province, 2015-2016. Chin J Vaccines Immunization. 2017;23(1):18-21+ 12

37. Liu W. Study on economic burden of bacterial meningitis in China. Beijing Chinese Center for Disease Control and Prevention; 2016
38. Lanzhou Institute of Biological Products Co. L. Instruction manual for Haemophilus influenzae type b conjugate vaccine. http://www.vacmic.com/ htm/20163/13_991.htm. Accessed 08 Jan 2021.

39. Lai $X$, Rong H, Ma X, Hou Z, Li S, Jing R, et al. Willingness to pay for seasonal influenza vaccination among children, chronic disease patients, and the elderly in china: a national cross-sectional survey. Vaccines (Basel). 2020;8(3).

40. Zhuang JL, Wagner AL, Laffoon M, Lu YH, Jiang QW. Procurement of category 2 vaccines in China. Vaccines (Basel). 2019;7(3).

41. WHO Commission on Macroeconomics and Health, WHO. Macroeconomics and health: investing in health for economic development: executive summary/report of the Commission on Macroeconomics and Health. Geneva: World Health Organization; 2001. http://www.who.int/iris/handle/1 0665/42463. Accessed 05 May 2021

42. Woods B, Revill P, Sculpher M, Claxton K. Country-level cost-effectiveness thresholds: initial estimates and the need for further research. Value Health. 2016;19:929-35

43. Ning G, Yin Z, Li Y, Wang H, Yang W. Cost-effectiveness of the Haemophilus influenzae type $b$ vaccine for infants in mainland China. Hum Vaccin Immunother. 2018;14(1):36-44.

44. Pan XF, Yang CX, Mark J, Ulla KG, Clark A, Pennington M. Economic evaluation of including Haemophilus influenzae type $b$ vaccine in the national Expanded Program on Immunization in China. In: 2015 Annual Meeting of Sichuan University Doctorate Academic Forum Sichuan. China: Sichuan University; 2015. p. 4835-47.

45. Zheng Y, Rodewald L, Yang J, Qin Y, Pang M, Feng L, et al. The landscape of vaccines in China: history, classification, supply, and price. BMC Infect Dis. 2018;18(1):502.

46. Feng XL, Theodoratou E, Liu L, Chan KY, Hipgrave D, Scherpbier R, et al. Social, economic, political and health system and program determinants of child mortality reduction in China between 1990 and 2006: A systematic analysis. J Glob Health. 2012;2(1):010405.

\section{Publisher's Note}

Springer Nature remains neutral with regard to jurisdictional claims in published maps and institutional affiliations.
Ready to submit your research? Choose BMC and benefit from:

- fast, convenient online submission

- thorough peer review by experienced researchers in your field

- rapid publication on acceptance

- support for research data, including large and complex data types

- gold Open Access which fosters wider collaboration and increased citations

- maximum visibility for your research: over $100 \mathrm{M}$ website views per year

At BMC, research is always in progress.

Learn more biomedcentral.com/submissions 